\title{
Highly Accurate Online Characterisation of Cracks in Plate-Like
}

\author{
Structures \\ Fangxin Zou ${ }^{\mathrm{a}, 1}$, Jing Rao ${ }^{\mathrm{b}}$, M. H. Aliabadi ${ }^{\mathrm{a}}$ \\ ${ }^{\text {a }}$ Department of Aeronautics, Imperial College London, South Kensington Campus, \\ London, SW7 2AZ, UK \\ ${ }^{\mathrm{b}}$ School of Mechanical and Aerospace Engineering, Nanyang Technological \\ University, 50 Nanyang Avenue, Singapore
}

\begin{abstract}
In this paper, an in situ technique for the localisation and the sizing of cracks in plate-like structures is presented. The excitation and the reception of the ultrasonic diagnostic signal - Lamb waves - are achieved by permanently installed piezoelectric ceramic disks. The technique simplifies the underdetermined problem of the full characterisation of cracks to an over-determined problem of the localisation of crack tips. Also, it overcomes the difficulties associated with using the pitch-catch excitation scheme for crack characterisation. The development of the technique was done systematically using analytic models and the finite element method. The technique has been validated by an experiment and assessed by extensive parametric studies. While it was designed to be a convenient approach, highly accurate crack characterisation has been attained as evident through the results of both numerical studies and physical experiments.
\end{abstract}

Keywords: non-destructive evaluation, structural health monitoring, crack detection, piezoelectric transducers, Lamb waves, multilateration

\footnotetext{
${ }^{1}$ Corresponding author. Email addresses: f.zou11@imperial.ac.uk (F. Zou), jrao001@e.ntu.edu.sg (J. Rao).
} 


\section{Introduction}

The importance of structural integrity to critical engineering structures such as aircrafts and pressure vessels cannot be exaggerated. The condition inspection of these structures still very much depends on conventional non-destructive evaluation (NDE) techniques such as ultrasonic C-scanning and X-ray scanning. The execution of these techniques requires structures to be pulled out of service and hence incurs significant costs. More importantly, due to the extreme loading conditions that it is subjected to, a critical engineering structure may encounter failure before it is due for a scheduled inspection.

In recent years, the concept of online structural health monitoring (SHM) has attracted much attention and research effort. In particular, it has been shown that Lamb waves can be used effectively for the detection of defects in plate-like structures. The excitation and the reception of Lamb waves can be achieved efficiently by compact piezoelectric patches. To date, the types of defects, which Lamb waves have been able to detect, include, but are not limited to cracks [1-3], holes $[4,5]$ and delaminations $[6,7]$. However, due to the underdetermined nature of the inverse problem, techniques for the sizing of cracks without a priori knowledge of locations have rarely been reported. Although phased array scanning has demonstrated such potential [8], it requires significantly large numbers of transducers and, yet, still struggles to characterise cracks that are not perpendicular to the purposely formed wave beams.

The technique presented in this paper fills in the abovementioned knowledge gap in the development of SHM. It utilises piezoelectric disks that are excited sequentially in the pulse-echo scheme. The pulse-echo excitation scheme, which processes backscattered signals that are reflected off the features of interest, has been well studied and exploited for NDE [9]. For the detection of cracks on plate-like structures, it will not lead 
to situations in which an actuator-sensor path intersects with a crack and hence outputs signals that give ambiguous information about the crack, as the pitch-catch excitation scheme will do. The detection and the sizing of cracks can be represented by the localisation of crack tips which are omni-directional scattering sources. If carefully executed, this can become a determined or even an over-determined problem. The signal features of interest are the time-of-flight's (ToFs) of the echoes from crack tips. The localisation of the tips of a crack is made possible by a two-stage approach. At first, the method of triangulation is used to provide the initial estimates of locations. Then, these estimates are further enhanced by a multilateration (MLAT) algorithm [10] and the removal of the sensor signals that involve interactions with the crack line.

The development of the technique made use of both analytic models and the finite element method (FEM). The analytic models were used for the tuning of diagnostic signal, the FEM was employed to simulate the propagation of Lamb waves. The high accuracy of the technique in crack characterisation has been validated by an experiment. A series of parametric studies that were based on numerical simulations have also been carried out in order to obtain a comprehensive understanding of the performance of the technique. 


\section{Development of Detection Strategy}

\subsection{Specimens}

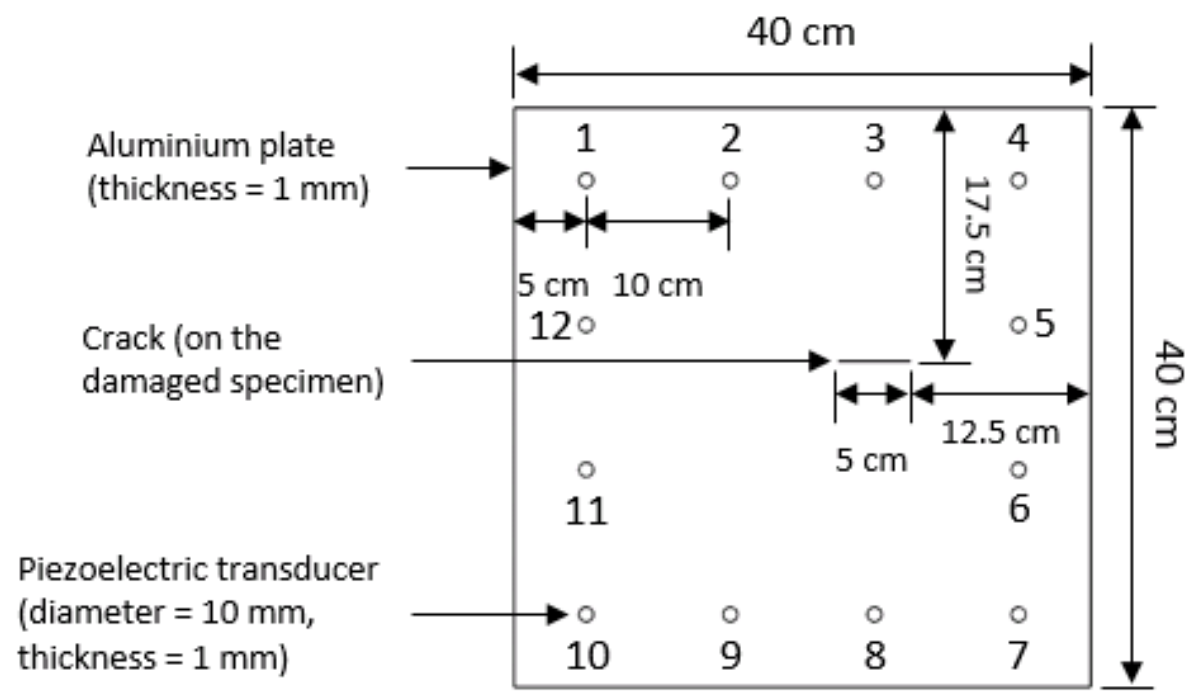

Figure 1 Schematic diagram of the specimens used for the development of the technique (the transducers are numbered from 1 to 12 clockwise starting from the top left corner).

Table 1 Properties of the materials used for the specimens.

\begin{tabular}{|l|c|c|}
\hline \multicolumn{2}{|c|}{ Aluminium } & Piezoelectric Ceramic \\
\hline Density $\left(\mathbf{k g} / \mathbf{m}^{\mathbf{3}}\right)$ & 2700 & 7800 \\
\hline Young's modulus (GPa) & 70 & 100 \\
\hline Poisson's ratio & 0.33 & 0.33 \\
\hline
\end{tabular}

The development of the technique is demonstrated using the pristine and the damaged specimen illustrated in Figure 1. On each of the specimens, a network of 12 piezoelectric disks are permanently adhered. The area bounded by the piezoelectric disks is referred to 
as the effective detection area. It is expected that any crack within this area will be detectable.

The material properties of the aluminium plate and the piezoelectric disks are given in Table 1. Since the current technique does not take into account signal amplitudes, the electric and the piezoelectric properties of the transducers do not need to be considered.

\subsection{Tuning of Diagnostic Signal}

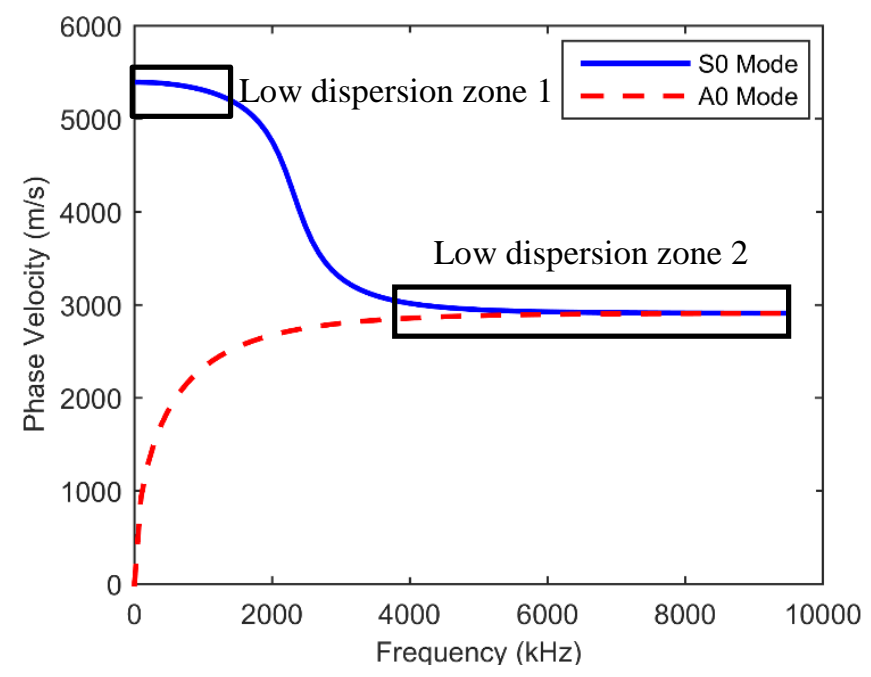

Figure 2 Dispersion curves of the $\mathrm{S} 0$ and the A0 Lamb wave mode in a $1 \mathrm{~mm}$ thick aluminium plate.

The dispersion curves of the two types of Lamb wave modes - the symmetric (S) and the anti-symmetric (A) mode - can be found by solving the Rayleigh-Lamb equations [11]. In Figure 2, the dispersion curves of the two fundamental Lamb wave modes - the S0 and the A0 mode - in the aluminium plate shown in Figure 1 are displayed.

The responses of a plate that is excited by a piezoelectric actuator have been studied by many [12-15]. At a certain frequency, the response of a certain Lamb wave 
mode (S0, A0, S1, A1, S2, A2, etc.) to the excitation induced by a piezoelectric disk is given by [15]

$$
u(r)=i C a J_{1}(k a) \frac{N(k)}{D^{\prime}(k)} H_{1}^{(2)}(k r)
$$

where for $\mathrm{S}$ modes,

$$
\begin{gathered}
N(k)=k q\left(k^{2}+q^{2}\right) \cos (p d) \cos (q d) \\
D(k)=\left(k^{2}-q^{2}\right)^{2} \cos (p d) \sin (q d)+4 k^{2} p q \sin (p d) \cos (q d)
\end{gathered}
$$

and for A modes,

$$
\begin{gathered}
N(k)=k q\left(k^{2}+q^{2}\right) \sin (p d) \sin (q d) \\
D(k)=\left(k^{2}-q^{2}\right)^{2} \sin (p d) \cos (q d)+4 k^{2} p q \cos (p d) \sin (q d)
\end{gathered}
$$

in which

$$
\begin{aligned}
& p=\sqrt{\frac{\omega^{2}}{c_{L}^{2}}-k^{2}} \\
& q=\sqrt{\frac{\omega^{2}}{c_{T}^{2}}-k^{2}}
\end{aligned}
$$

In equation (1), $r$ is the radial position at where the displacement is calculated, $\omega$ is the circular frequency, $k$ is the wavenumber of the corresponding Lamb wave mode, $a$ is the radius of the piezoelectric disk, $d$ is the half-thickness of the plate, $C$ is a constant that is determined by the property of the bonding layer, $c_{L}$ and $c_{T}$ are the velocities of the bulk waves, $J_{1}$ is the Bessel function of the first kind and order unity, and $H_{1}^{(2)}$ is the Hankel 
function of the second kind and order unity. For the aluminium plate shown in Figure 1, the values of $c_{L}$ and $c_{T}$ are $6197.8 \mathrm{~m} / \mathrm{s}$ and $3122.0 \mathrm{~m} / \mathrm{s}$ respectively.

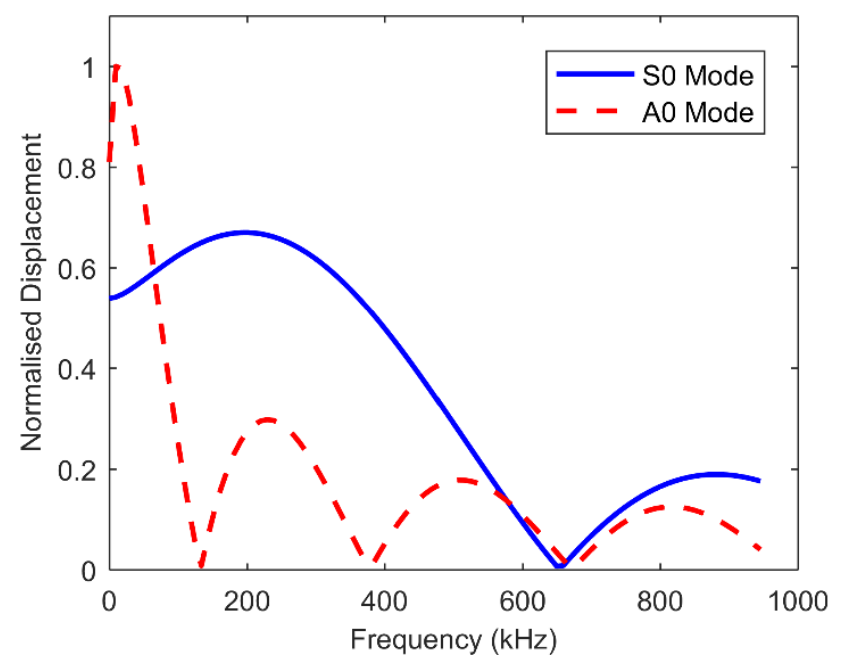

Figure 3 Displacements of the S0 and the A0 Lamb wave mode that are excitable at different frequencies by a piezoelectric disk with a diameter of $10 \mathrm{~mm}$ in a $1 \mathrm{~mm}$ thick aluminium plate.

In Figure 3, the frequency-dependent displacements of the S0 and the A0 mode that can be excited in the specimens shown in Figure 1 are plotted. At certain frequencies, the response of one of the two modes diminishes.

From Figure 2, it can be seen that the S0 mode is less dispersive than the A0 mode at low frequencies. Also, it is able to deliver stronger reflections from cracks [16]. Therefore, in this work, it is desirable to excite the piezoelectric disks at frequencies at which the response of the A0 mode is minimal. As observed in Figure 3, both the frequencies of 130 and $380 \mathrm{kHz}$ satisfy this requirement. When transducers are being excited in the pulse-echo scheme, they will not be able to capture any reflected signals [17]. Therefore, the higher frequency of $380 \mathrm{kHz}$ is chosen due to the shorter wavelength and hence the smaller blind detection zones which it will lead to. 
Hanning-windowed sinusoidal tonebursts are used as the diagnostic signals in this work. Since only ToFs are of interest, these tonebursts are assigned with three cycles in order to result in relatively short wavelengths. By doing so, blind detection zones will be minimised, and different wave packets in sensor signals will be more separated and distinguishable.

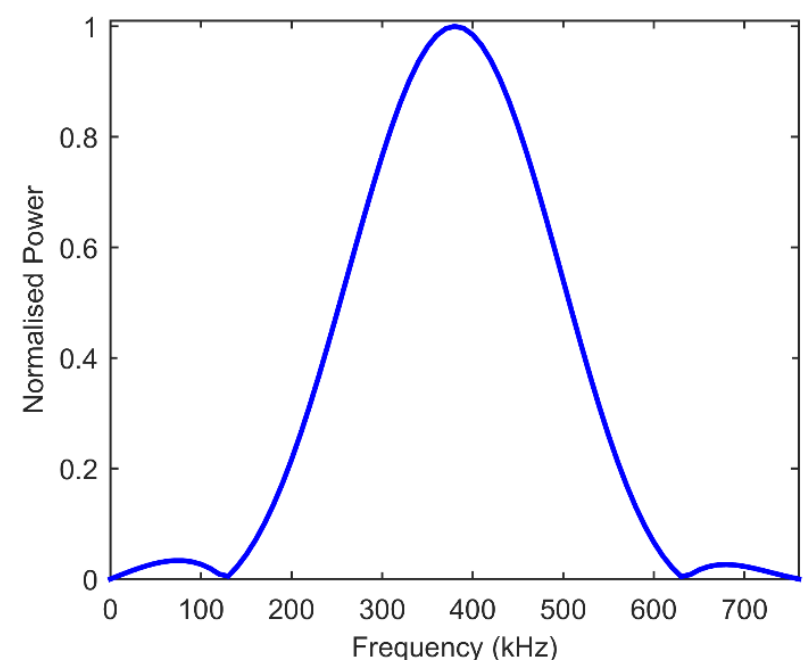

Figure 4 Frequency spectrum of a three-cycle Hanning-windowed sinusoidal toneburst with a central frequency of $380 \mathrm{kHz}$.

The frequency spectrum of the diagnostic signals is displayed in Figure 4. Since Lamb waves are dispersive such that different frequency components propagate at different velocities, the waveforms of excited and received signals may be different. Nevertheless, it can be seen from Figure 2 that all of the frequency components of the diagnostic signals lie well within the first low dispersion zone of the S0 mode. Therefore, changes in waveform will be insignificant.

\subsection{Finite Element Modelling}

The FEM has been widely used for modelling the propagation of Lamb waves [18-21]. In this work, the development of the technique relies on the FEM for simulating the 
responses of the specimens under piezoelectric excitation. Finite element simulations are carried out by the commercial FEM package Abaqus®/Explicit. In the finite element models, the aluminium plates are formulated by 4-node conventional shell elements (S4R) and the piezoelectric disks are formulated by 8-node 3D stress elements (C3D8R). In a previous study [6], it is shown that when shells are assigned as the mid-planes of plates, the best agreement between numerical and experimental results will be obtained. Shell elements and the bottom surfaces of piezoelectric disks are set apart by the half-thickness of the plate that is modelled. The plate and the bottom surfaces of the piezoelectric disks are perfectly coupled via the surface-to-surface tie constraint which equates the strains that are interpolated over the two connected surfaces. On the damaged specimen, the crack is modelled as a seam, along which all the nodes are disconnected.

The excitation and the reception of Lamb waves are modelled according to the scheme introduced by Yang et al [22]. In the simulations, piezoelectric actuators are applied with radial displacements at the circumferences of their bottom surfaces, and sensor signals are obtained by averaging the nodal strain values of the bottom surfaces of sensors. Since the amplitudes of sensor signals are not of interest, the amplitudes of the displacements to be applied to actuators do not matter so much as long as they do not induce any numerical instability.

The accuracy of a finite element model largely depends on the level of mesh convergence. For a dynamic analysis, due to the time dispersion effect, the time period to be considered in the mesh convergence study must be equal to or greater than the duration of the actual analysis. In this work, the mesh convergence study is performed by making use of the longest actuator-sensor path (i.e. one of the diagonal paths) on the pristine 
specimen. The duration of the analysis is set to the time required by the diagnostic signal for making a return trip along this path.

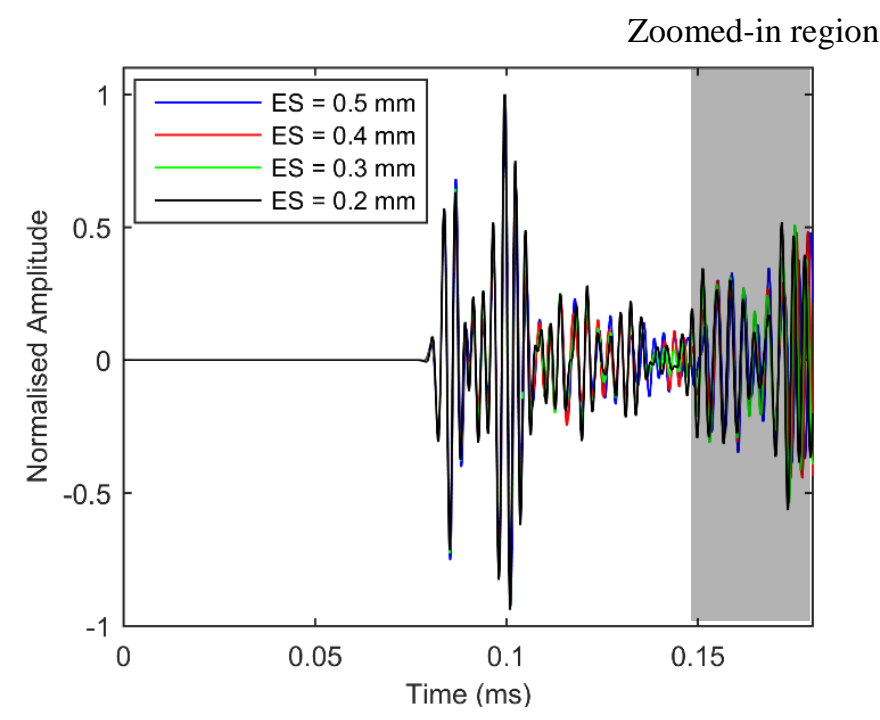

(a)

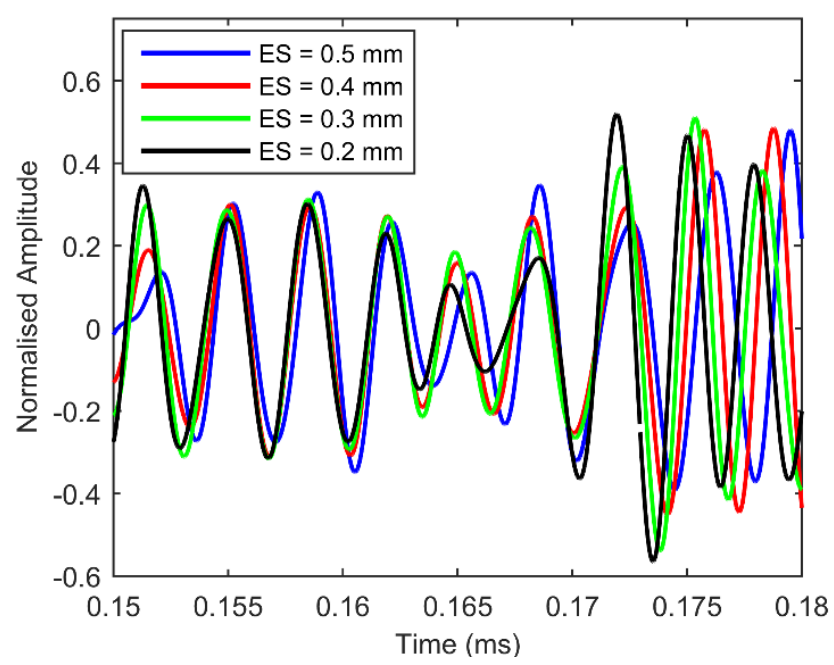

(b)

Figure 5 Result of the mesh convergence study: (a) full-scale time histories and (b) zoomed-in time histories (ES stands for element size).

Figure 5 shows the mesh convergence of the signal captured by Transducer 7 when Transducer 1 is used as the actuator. As element sizes decrease, signal amplitudes 
increase and phases shift forward. When the element size is $0.2 \mathrm{~mm}$, the level of mesh convergence remains satisfactory until approximately $0.165 \mathrm{~ms}$. However, this mesh could not be further refined since the resultant computational expenses would become unaffordable even for the high performance computers that were used for this work. In fact, as long as a crack is not extremely close to an edge of the effective detection area, the accuracy of the mesh with $0.2 \mathrm{~mm}$ elements will be sufficient.

\subsection{Calibration of Lamb Wave Velocity and Distance Offset}

Although the theoretical velocities of Lamb waves can be calculated from the RayleighLamb equations, the ones that are computed by numerical methods are often different and vary with modelling parameters. In the case of the FEM, these modelling parameters include, but are not limited to, mesh size and time increment. An equivalent analogy in reality could be when the actual properties of the materials used are different from the stated values. Also, uncertainties, such as delays in instrumentation, will cause offsets in distance measurements. In this work, it is found that these offsets, which were often neglected $[4,23]$, play a crucial role in determining the locations of crack tips. Therefore, it is essential to calibrate for the velocity of Lamb waves and the offset in distance for every combination of structure and hardware setup. 


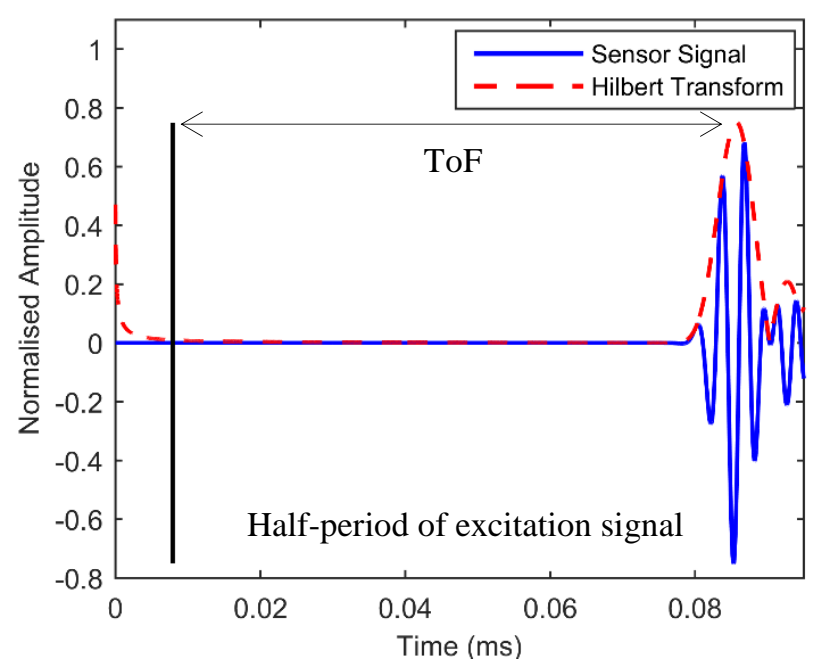

Figure 6 Procedure for determining ToFs.

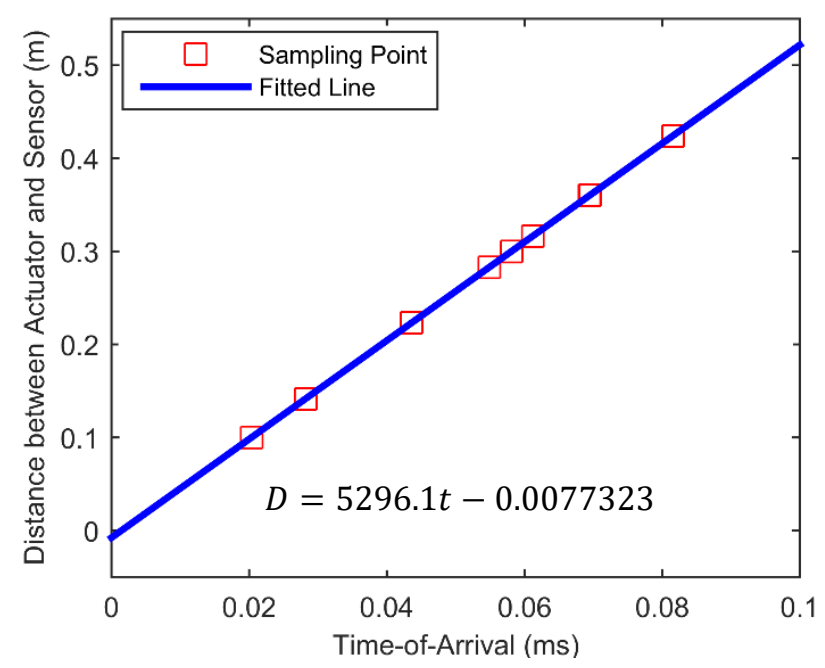

Figure 7 Calibration of the velocity of Lamb waves and the offset in distance.

The calibration of the velocity of Lamb waves and the offset in distance is carried out using all of the distinctive actuator-sensor paths on the pristine specimen. The calculation of the velocity of Lamb waves for a certain path depends on the distance between the actuator and the sensor and the ToF of the sensor signal. As illustrated in Figure 6 , in the current technique, the ToF of a sensor signal refers to the difference in time between the half-period of the excitation signal and the time instant at which the first 
peak of the Hilbert transform of the sensor signal arrives. Figure 7 displays the calibration data of the specimens shown in Figure 1.

\subsection{Triangulation}

The method of triangulation is used to provide initial estimates of the locations of crack tips. In the current technique, the transducers are excited sequentially, and every transducer is responsible for both the excitation and the reception of Lamb waves. When Lamb waves encounter a crack tip, they will be scattered omni-directionally [24]. The waves that travel from a crack tip back to a transducer can be obtained by subtracting the sensor signal acquired on the pristine plate from that acquired on the damaged plate. It is assumed that in every back scattered signal, the first wave packet represents the reflection off the crack tip that is closest to the transducer by which the signal is received.
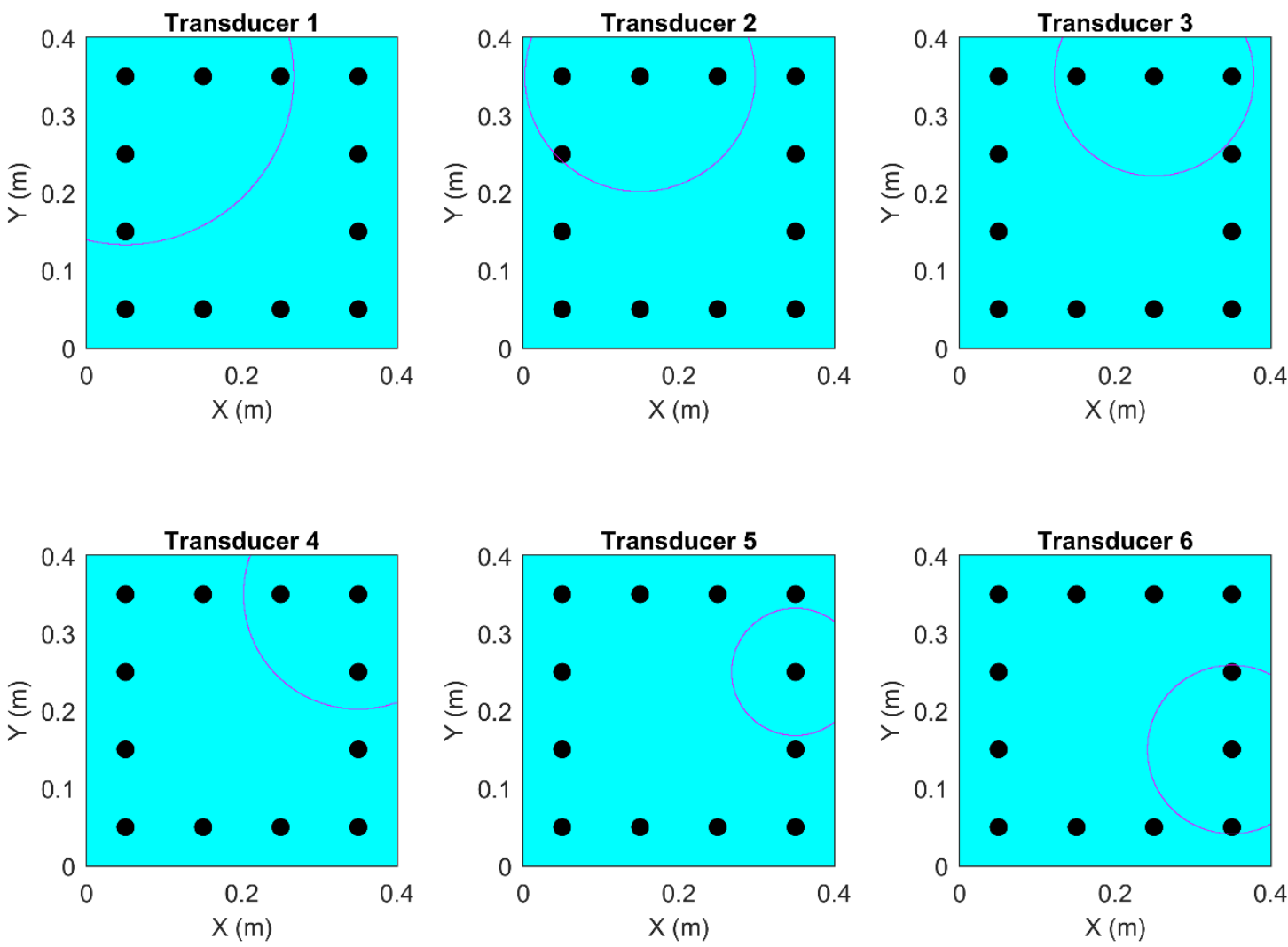

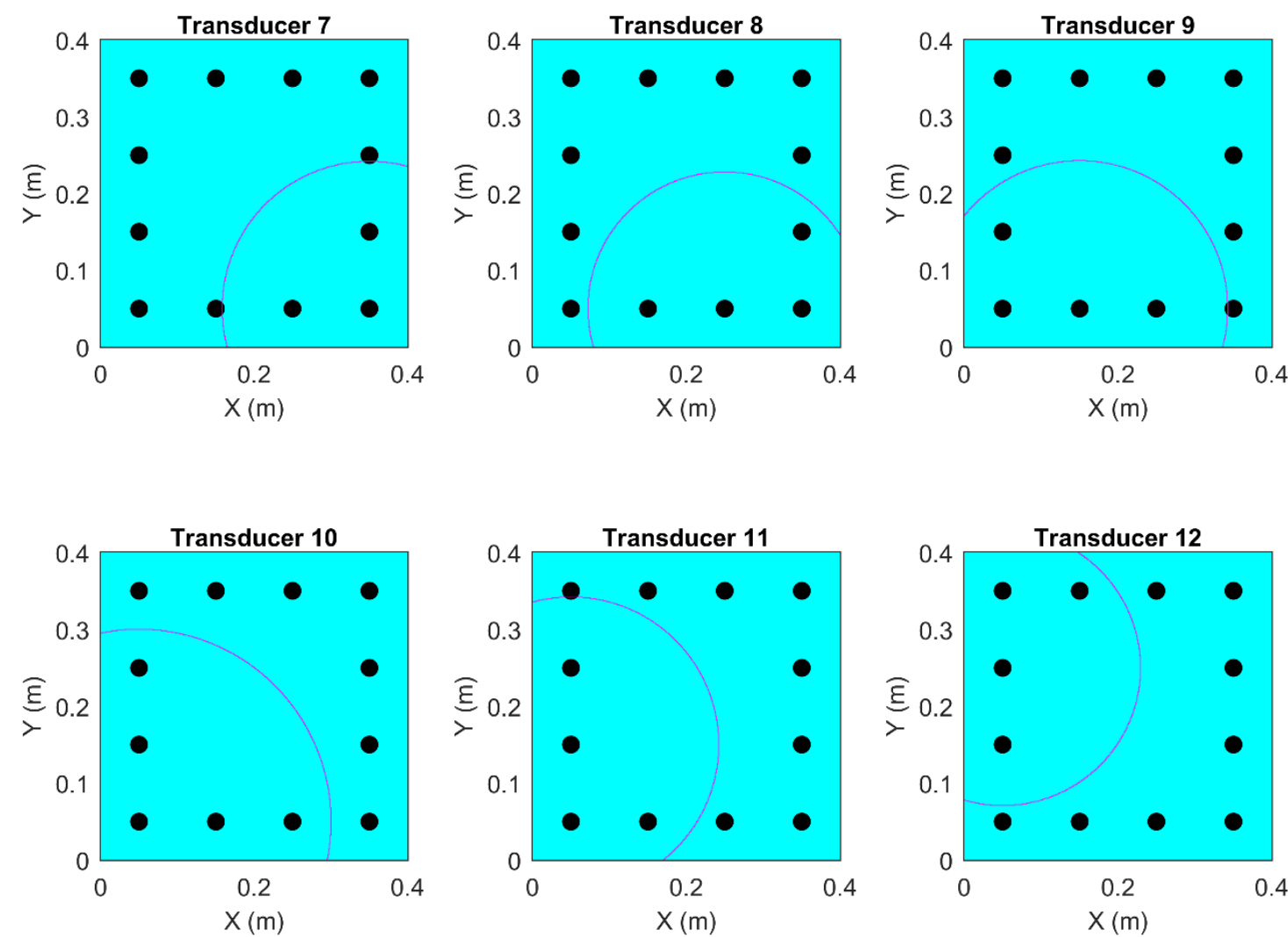

Figure 8 Detection locus of each transducer.

For each transducer, a locus of circular shape, which covers all of the possible locations of its nearest crack tip, can be constructed based on the ToF of the first wave packet of the back scattered waves using the relationship

$$
T o F=\frac{2\left(\sqrt{\left(x^{c t}-x^{t}\right)^{2}+\left(y^{c t}-y^{t}\right)^{2}}-c_{c a l}\right)}{v_{c a l}}
$$

where $v$ is the velocity of Lamb waves, $c$ is the offset in distance, the subscript cal means calibration data, and the superscripts $c t$ and $t$ stand for crack tip and transducer. Figure 8 plots the detection loci that are associated with the transducers on the specimens shown in Figure 1. These images are generated by firstly dividing the specimens into pixels, and 
then assigning to each pixel the identification number of the transducer whose associated locus satisfies equation (2).

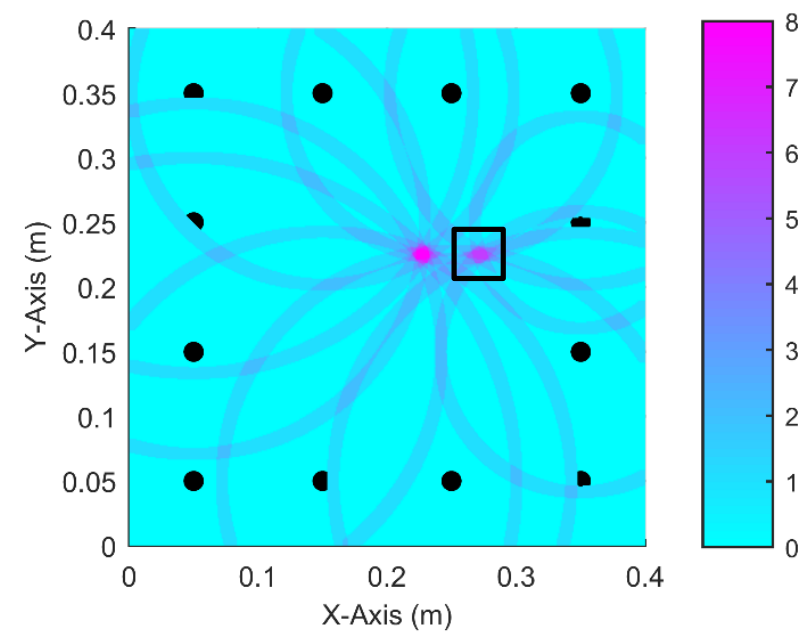

Figure 9 Number of the detection loci for the first crack tip.

For each pixel, the number of the detection loci that exist within the surrounding area is determined. The choice of the dimension of the surrounding area is determined by both the expected crack length and the desired detection accuracy. If the surrounding area is too large, different crack tips would not be distinguishable from each other, and if it is too small, an excessive number of crack tips would be identified. The latter case is due to the fact that each transducer may exhibit an offset in distance that differs slightly from the calibration data, and hence detection loci that correspond to the same crack tip might intersect at more than one point. The dimension of the surrounding area used in this work is $1 \mathrm{~cm} \times 1 \mathrm{~cm}$. Figure 9 shows a map of the numbers of detection loci. The crack tip that is detected by the largest number of loci can be easily identified. 


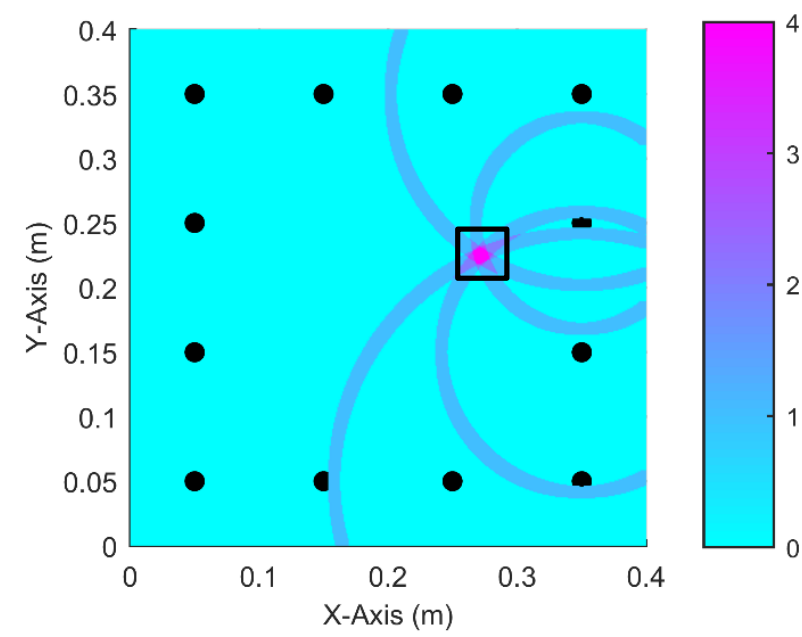

Figure 10 Number of the detection loci for the second crack tip.

Table 2 Crack parameters estimated by the initial triangulation.

Triangulation Actual Error (\%)

\begin{tabular}{lllll} 
Crack Tip 1 & $\mathbf{x}$ & 0.22772 & 0.225 & 5.4490 \\
\hline (m) & $\mathbf{y}$ & 0.22529 & 0.225 & 0.57143 \\
\hline Crack Tip 2 & $\mathbf{x}$ & 0.27169 & 0.275 & 6.6271 \\
\hline (m) & $\mathbf{y}$ & 0.22486 & 0.225 & 0.28814 \\
\hline Crack Centre & $\mathbf{x}$ & 0.24971 & 0.25 & 0.58907 \\
\hline (m) & $\mathbf{y}$ & 0.22507 & 0.225 & 0.14165 \\
\hline Crack Length (m) & & 0.043964 & 0.05 & 12.072 \\
\hline Crack Orientation $\left({ }^{\circ}\right)$ & -0.56012 & 0 & N/A
\end{tabular}


Although the existence of another crack tip is also visible in Figure 10, the loci, which detect the first crack tip, are to be removed from the figure in order to obtain a clearer image. In Figure 10, the existence of the second crack tip is made more obvious. If there are more than two crack tips in a plate, the removal of detection loci would continue until all crack tips have been identified. The result of the initial triangulation is given in Table 2. The estimated locations of the crack tips are found by averaging the coordinates of the pixels with the highest number of detection loci. The percentage errors are calculated by normalising the crack parameters (except for crack orientation) against the actual crack length.

\subsection{Multilateration Algorithm}

A nonlinear least squares based iterative MLAT algorithm, which is adopted from the field of navigation [10], is used to improve the initial estimates of the locations of crack tips. One of the key features of the MLAT algorithm is that it is capable of solving overdetermined systems of equations to obtain the most accurate solutions. In the context of this work, the parameters to optimise are

$$
\boldsymbol{x}_{i}=\left[\begin{array}{llll}
x_{i}^{c t} & y_{i}^{c t} & v_{i} & c_{i}
\end{array}\right]^{T}
$$

The improvement of initial estimates is done for one crack tip at a time, starting with the crack tip that is detected by the largest number of transducers. In every iteration of the MLAT algorithm, the residual between the observed and the calculated ToFs is found by

$$
\Delta \boldsymbol{x}_{i}=\left(\boldsymbol{H}^{T} \boldsymbol{H}\right)^{-1} \boldsymbol{H}^{T}\left(\boldsymbol{T o F}^{o}-\boldsymbol{T o F}_{i}^{c}\right)
$$

where 


$$
\begin{aligned}
& \boldsymbol{T o F}_{i}^{c}=\left[\begin{array}{c}
\frac{2\left(\sqrt{\left(x_{i}^{c t}-x_{1}^{c t}\right)^{2}+\left(y_{i}^{c t}-y_{1}^{c t}\right)^{2}}-c_{i}\right)}{v_{i}} \\
\frac{2\left(\sqrt{\left(x_{i}^{c t}-x_{2}^{c t}\right)^{2}+\left(y_{i}^{c t}-y_{2}^{c t}\right)^{2}}-c_{i}\right)}{v_{i}} \\
\vdots \\
\frac{2\left(\sqrt{\left(x_{i}^{c t}-x_{N}^{c t}\right)^{2}+\left(y_{i}^{c t}-y_{N}^{c t}\right)^{2}}-c_{i}\right)}{v_{i}}
\end{array}\right] \\
& \boldsymbol{H}=\left[\begin{array}{cccc}
\frac{d T o F_{1}^{c}}{d x^{c t}} & \frac{d T o F_{1}^{c}}{d y^{c t}} & \frac{d T o F_{1}^{c}}{d v} & \frac{d T o F_{1}^{c}}{d c} \\
\frac{d T o F_{2}^{c}}{d x^{c t}} & \frac{d T o F_{2}^{c}}{d y^{c t}} & \frac{d T o F_{2}^{c}}{d v} & \frac{d T o F_{2}^{c}}{d c} \\
\vdots & \vdots & \vdots \\
\frac{d T o F_{N}^{c}}{d x^{c t}} & \frac{d T o F_{N}^{c}}{d y^{c t}} & \frac{d T o F_{N}^{c}}{d v} & \frac{d T o F_{N}^{c}}{d c}
\end{array}\right]
\end{aligned}
$$

In equations (5) and (6), $N$ is the number of the transducers that detect the crack tip. The residual is then added to the parameters of the current iteration to form those of the next iteration such that

$$
x_{i+1}=x_{i}+\Delta x_{i}
$$

The iterative process stops when the residual is smaller than a pre-set tolerance.

For determining the location of each crack tip, the MLAT algorithm only considers the signals captured by the transducers that are closest to this crack tip. If a crack tip is detected by no less than four transducers, the velocity of Lamb waves and the offset in distance could also be re-evaluated. Otherwise, they would make use of the calibration data, and equations (3), (5) and (6) would become

$$
\boldsymbol{x}_{i}=\left[\begin{array}{ll}
x_{i}^{c t} & y_{i}^{c t}
\end{array}\right]^{T}
$$




$$
\begin{gathered}
\boldsymbol{T o F}_{i}^{c}=\left[\begin{array}{c}
\frac{2\left(\sqrt{\left(x_{i}^{c t}-x_{1}^{c t}\right)^{2}+\left(y_{i}^{c t}-y_{1}^{c t}\right)^{2}}-c_{c a l}\right)}{v_{c a l}} \\
\frac{2\left(\sqrt{\left(x_{i}^{c t}-x_{2}^{c t}\right)^{2}+\left(y_{i}^{c t}-y_{2}^{c t}\right)^{2}}-c_{c a l}\right)}{v_{c a l}} \\
\vdots
\end{array}\right] \\
\boldsymbol{H}=\left[\begin{array}{cc}
\left.\frac{d T o F_{1}^{c}}{\left.d x_{i}^{c t}-x_{N}^{c t}\right)^{2}+\left(y_{i}^{c t}-y_{N}^{c t}\right)^{2}}-c_{c a l}\right) & \frac{d T o F_{1}^{c}}{d y^{c t}} \\
\frac{d T o F_{2}^{c}}{d x^{c t}} & \frac{d T o F_{2}^{c}}{d y^{c t}} \\
\vdots & \vdots \\
\frac{d T o F_{N}^{c}}{d x^{c t}} & \frac{d T o F_{N}^{c}}{d y^{c t}}
\end{array}\right]
\end{gathered}
$$

The results of initial triangulations serve two purposes - providing the starting points for the iterations of the MLAT algorithm and identifying the transducers that are closer to each crack tip. In this example, it is identified that Transducers $1,2,9,10,11$ and 12 are closer to the first crack tip, and Transducers 4, 5, 6 and 7 to the second. Transducers 3 and 8 , on the other hand, seem to take part in detecting both crack tips. There are two possible reasons behind this observation. Firstly, since the offset in distance of each transducer could be slightly different from the calibration data, a locus, which in reality is only capable of detecting one crack tip, could look as if it also detects another. Secondly, the first wave packet in the signal captured by a transducer could very well be the reflection from a crack line rather than the scattering from a crack tip. Therefore, it will be a safe practice to neglect the information given by these two transducers in the MLAT algorithm.

Table 3 Crack parameters estimated by the MLAT algorithm. 


\section{MLAT Actual Error (\%)}

\begin{tabular}{lcccc} 
Crack Tip 1 & $\mathbf{x}$ & 0.22451 & 0.225 & 0.98248 \\
(m) & $\mathbf{y}$ & 0.22517 & 0.225 & 0.33859 \\
& & & & \\
Crack Tip 2 & $\mathbf{x}$ & 0.27555 & 0.275 & 1.0935 \\
\hline (m) & $\mathbf{y}$ & 0.22491 & 0.225 & 0.18061 \\
& & & & \\
Crack Centre & $\mathbf{x}$ & 0.25003 & 0.25 & 0.055498 \\
\hline (m) & $\mathbf{y}$ & 0.22504 & 0.225 & 0.078987 \\
& & & & \\
\hline Crack Length (m) & & 0.051039 & 0.05 & 2.0773 \\
\hline Crack Orientation $\left({ }^{\circ}\right)$ & -0.29143 & 0 & N/A
\end{tabular}

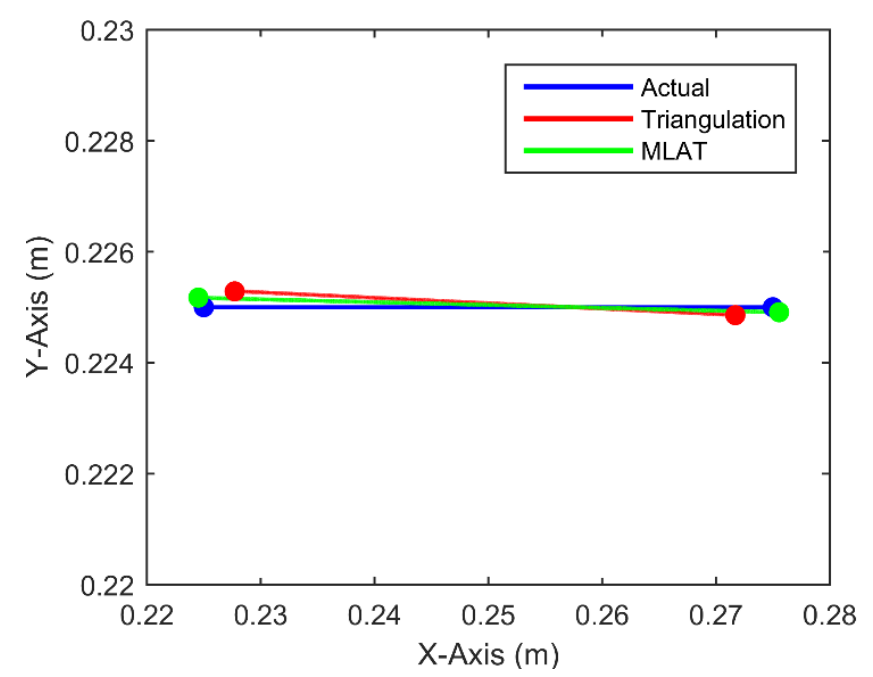

Figure 11 Comparison of the actual and the estimated crack lines.

Based on the estimated locations of the crack tips, it can be seen that Transducers 3 and 8 are closer to a point on the crack line than to any of the crack tips, suggesting that 
the first wave pack in each of the signals captured by these transducers is indeed the reflection from the crack line rather than the scattering from the closest crack tip. The examination of Tables 2 and 3 clearly indicates that the estimates of all crack parameters have been improved by the MLAT algorithm. In particular, the level of improvement in the estimate of the crack length, which is the most important parameter for assessing structural integrity, is significant (i.e. reduced from $12 \%$ to $2 \%$ ). The actual crack line and those estimated by the initial triangulation and by the MLAT algorithm are compared in Figure 11. The MLAT algorithm yields an improved result.

\section{Parametric Studies}

\subsection{Crack Length}

The sensitivity of current technique to crack length is firstly examined. This is done by varying the length of the crack on the damaged specimen while keeping the crack centre at the same position. In addition to the $5 \mathrm{~cm}$ crack, a $3 \mathrm{~cm}$ and a $1 \mathrm{~cm}$ crack are also considered. In theory, the transducers that detect each crack tip do not change.

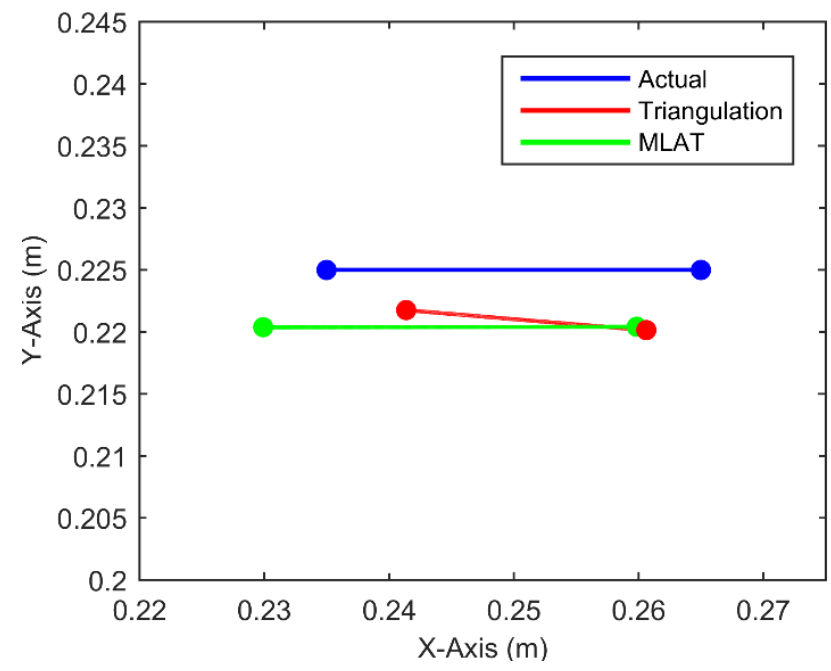

Figure 12 Comparison of the actual and the estimated lines of the $3 \mathrm{~cm}$ crack. 
The crack lines estimated by the initial triangulation and by the MLAT algorithm for the $3 \mathrm{~cm}$ crack are displayed in Figure 12. The most important parameter of a crack its length - is much better reconstructed by the latter approach. Based on the previous and the current example, it seems that initial triangulations tend to underestimate crack lengths. In fact, if the length of a crack cannot be sized with a 100\% accuracy, it would always be better, due to safety consideration, to overestimate it as the MLAT algorithm does.

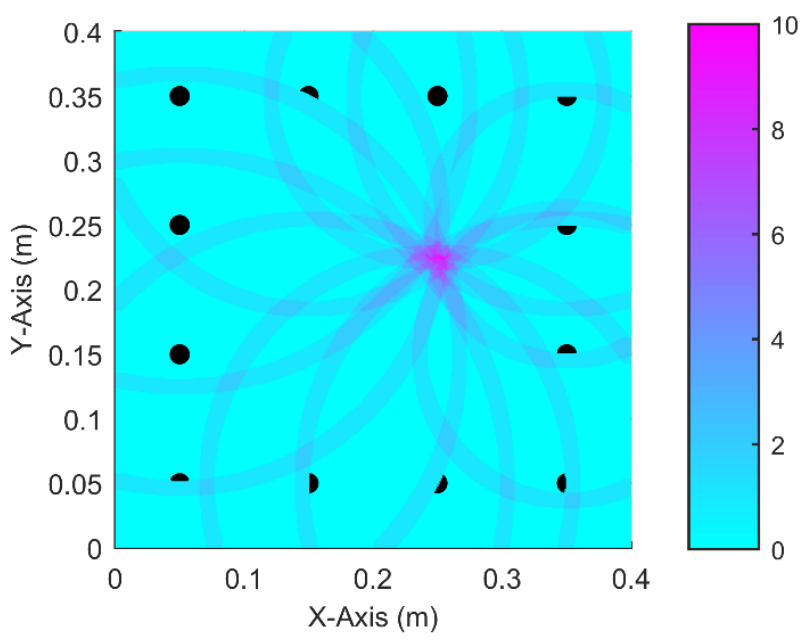

(a)

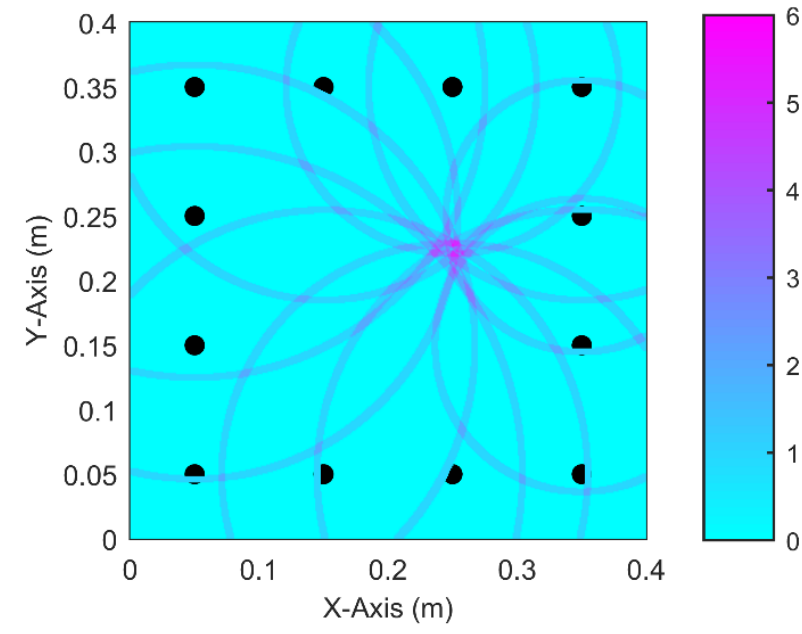

(b) 
Figure 13 Number of the detection loci for the first tip of the $1 \mathrm{~cm}$ crack. The plots were generated by considering a surrounding area of (a) $1 \mathrm{~cm} \times 1 \mathrm{~cm}$ and of (b) $0.5 \mathrm{~cm}$ $\times 0.5 \mathrm{~cm}$, within which detection loci were searched for.

Figure 13 shows the initial triangulation of a $1 \mathrm{~cm}$ crack. Although the existence of the crack is well detected by considering either surrounding area, in either case the crack cannot be effectively characterised. Comparison of the two figures shows that when a larger surrounding area is considered, the two crack tips become undistinguishable, and on the other hand, the consideration of a smaller surrounding area results in an excessive number of crack tips. This observation conforms with the prediction made in Section 2.5 about the choice of the dimension of surrounding area.

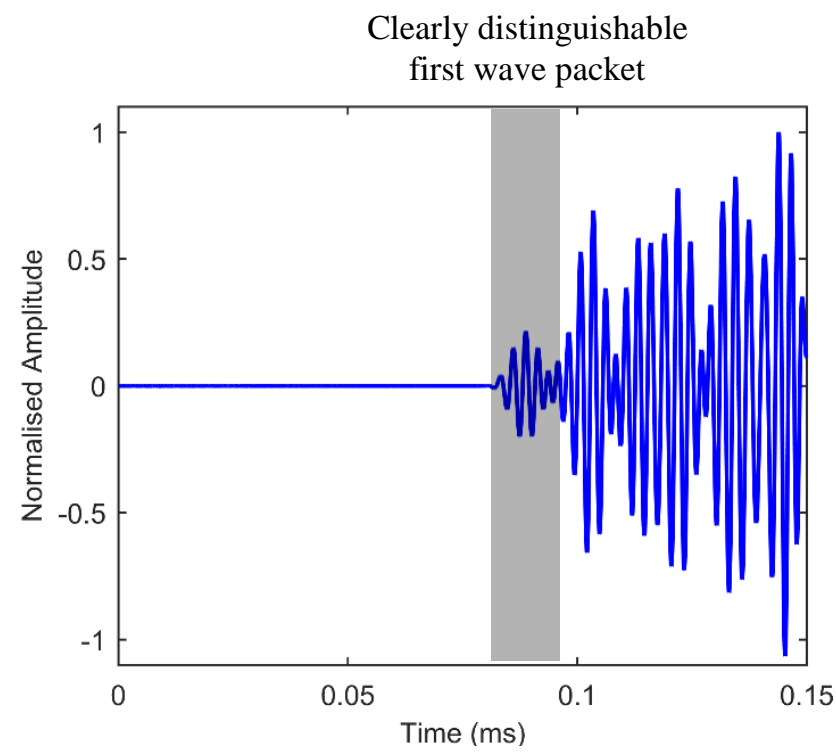

(a) 


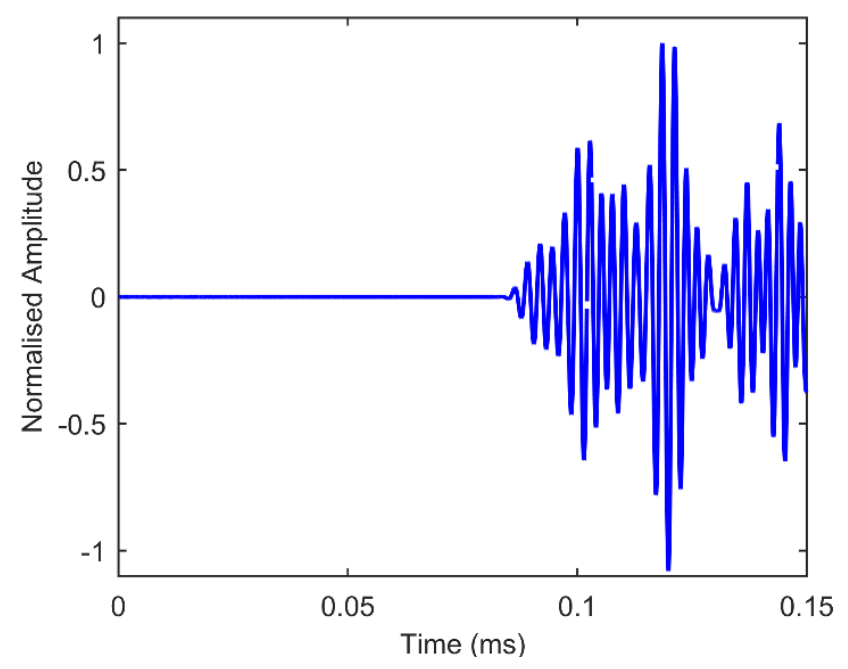

(b)

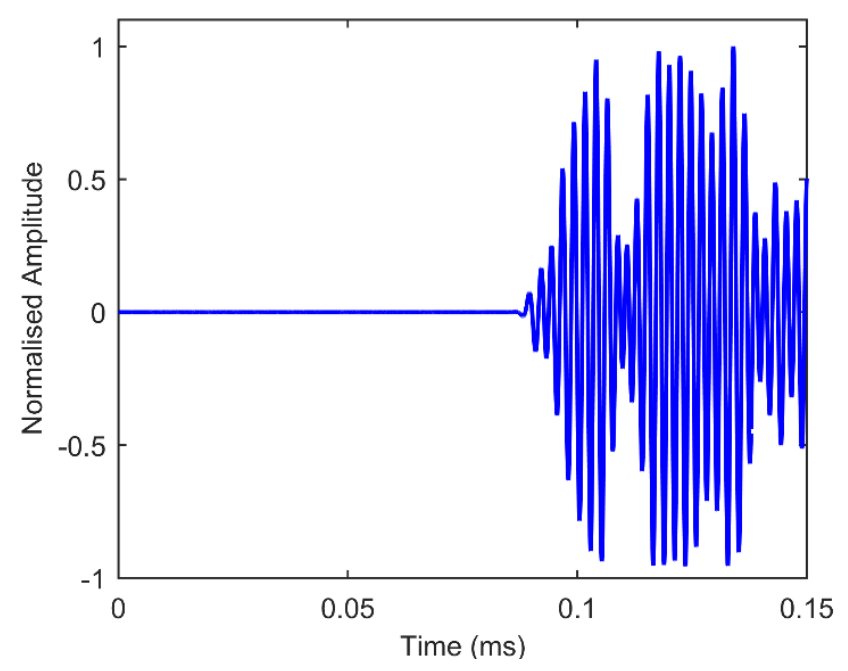

(c)

Figure 14 Back scattered waves captured by Transducer 1 from (a) the $5 \mathrm{~cm}$, (b) the 3 $\mathrm{cm}$ and (c) the $1 \mathrm{~cm}$ crack.

The lack of the capability of sizing the $1 \mathrm{~cm}$ crack could potentially be rectified by increasing the central frequency of the diagnostic signal. From Figure 14, it can be seen that as the length of a crack decreases, the first wave packet in the back scattered signal, which essentially originates from the closest crack tip, overlaps with the wave 
packets that come from the other features of the crack. Consequently, the determination of the ToF will be inaccurate. If the frequency of the diagnostic signal is increased, the first wave packet would become shorter in wavelength and hence more distinguishable again. The minimum crack length, which a diagnostic signal with a certain central frequency is capable of sizing, is commonly known as the resolution.

\subsection{Number of Transducers for Detection}

When both tips of a crack are detected by no less than four transducers, the MLAT algorithm would function without compromise. However, if one of the crack tips is detected by less than four transducers, the technique could be modified such that the determination of the location of the less illuminated crack tip by the MLAT algorithm makes use of the velocity of Lamb waves and the offset in distance that were obtained as the by-products of the determination of the location of the first crack tip.

In this section, the capability of the current technique in characterising cracks with one tip being detected by less than four transducers is assessed. The parameters of the crack on the damaged specimen is varied.

\subsubsection{Detection with Three Transducers}

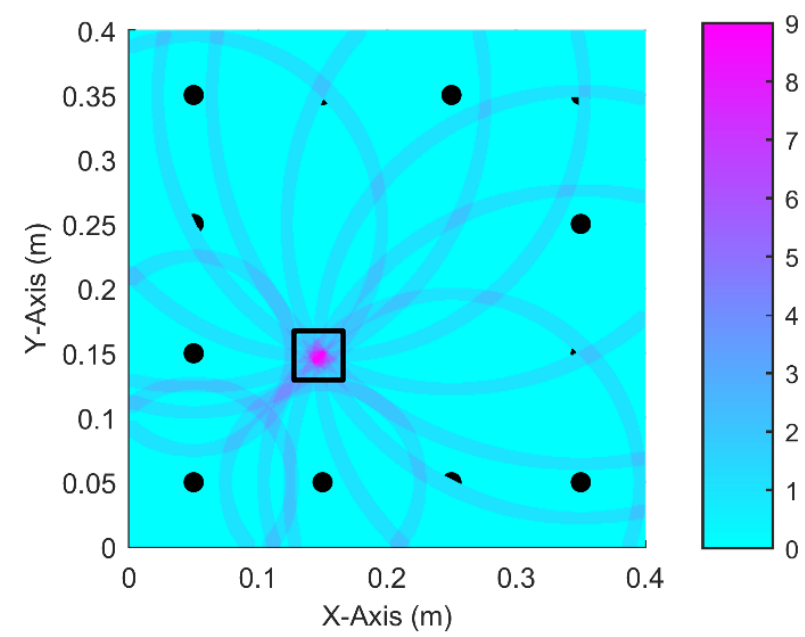


(a)

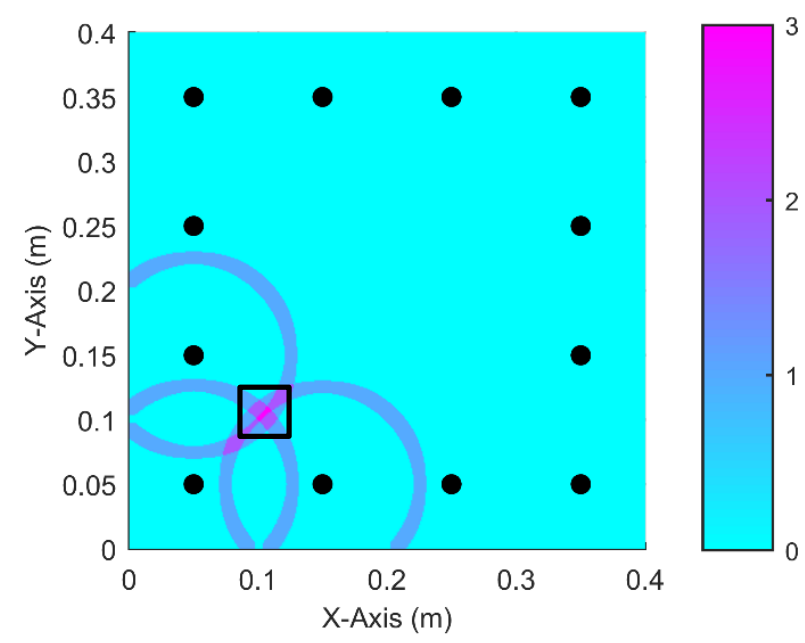

(b)

Figure 15 Number of the detection loci for (a) the first tip and (b) the second tip of the crack with one tip being detected by three transducers. The crack tips are located at $(0.1$ $\mathrm{m}, 0.1 \mathrm{~m})$ and $(0.15 \mathrm{~m}, 0.15 \mathrm{~m})$, and the crack is $7.07 \mathrm{~cm}$ long.

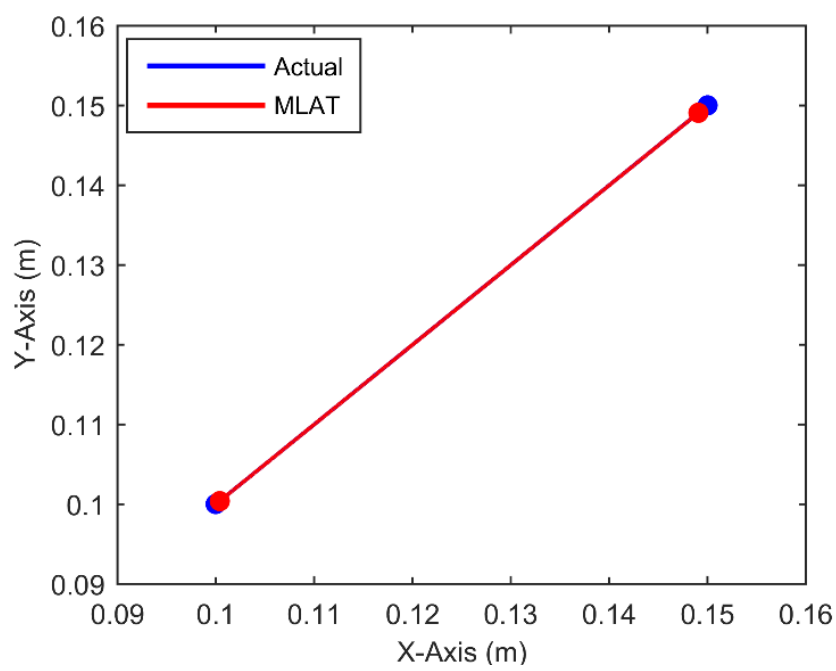

Figure 16 Comparison of the actual and the estimated lines of the crack with one tip being detected by three transducers. 
In Figure 9, both crack tips can be seen though the second crack tip is detected by a smaller number of transducers. However, in Figure 15(a), the existence of the second crack tip is not as visibly shown, and only becomes identifiable in Figure 15(b) after the removal of the loci that detect the first crack tip. This is due to the fact that in Figure 15(a), there are several other regions which contain just as many loci as the location of the second crack tip does. Figure 16 suggests that the characterisation of a crack, one of whose tips is seen by only three transducers, can still be done with a very high accuracy.

\subsubsection{Detection with Two Transducers}

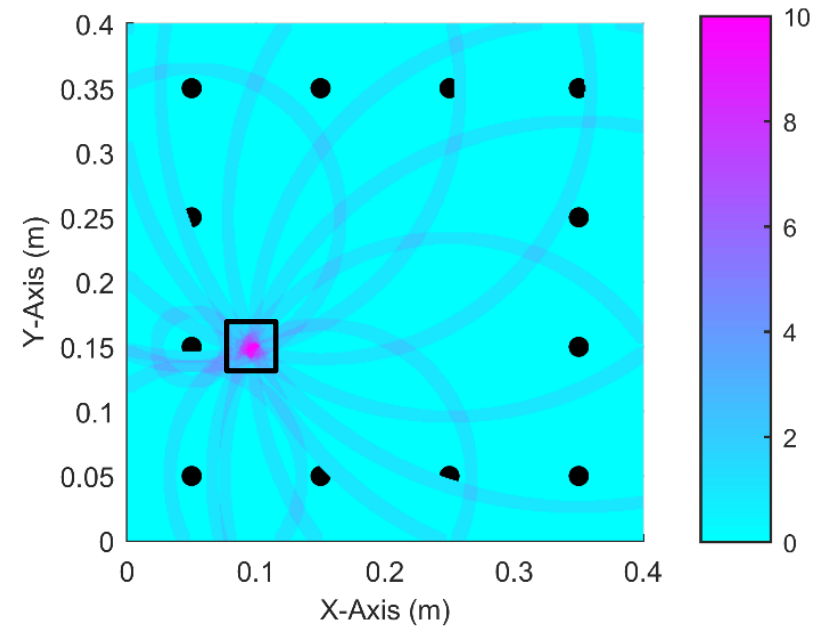

(a) 


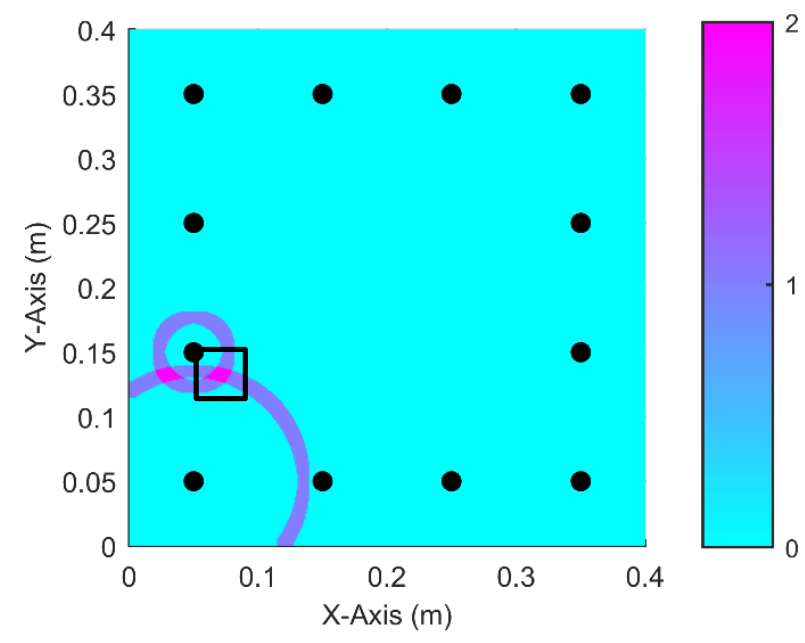

(b)

Figure 17 Number of the detection loci for (a) the first tip and (b) the second tip of the crack with one tip being detected by two transducers. The crack tips are located at $(0.06$ $\mathrm{m}, 0.13 \mathrm{~m})$ and $(0.1 \mathrm{~m}, 0.15 \mathrm{~m})$, and the crack is $4.47 \mathrm{~cm}$ long.

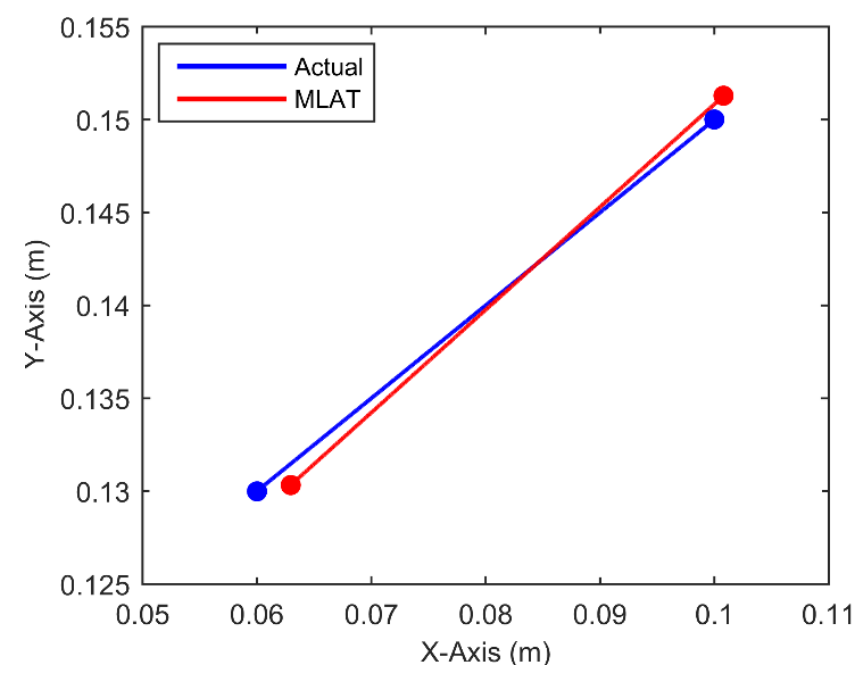

Figure 18 Comparison of the actual and the estimated lines of the crack with one tip being detected by two transducers.

In Figure 17(a), only the first crack tip is clearly identified. From Figure 17(b), it can be seen that the detection loci associated with Transducers 10 and 11 give rise to two regions 
in which the second crack tip could be located. Nevertheless, the one that is outside the effective detection area is merely a phantom due to symmetry [8]. If the second crack tip was really outside the effective detection area, Transducers 10 and 11 would rather be closer to a point on the crack line than to the second crack tip, and the detection loci associated with these transducers would intersect at different locations. As Figure 18 shows, with a little more consideration, a crack, one of whose tips is seen by only two transducers, can still be very well characterised.

\subsection{Kinked Cracks}

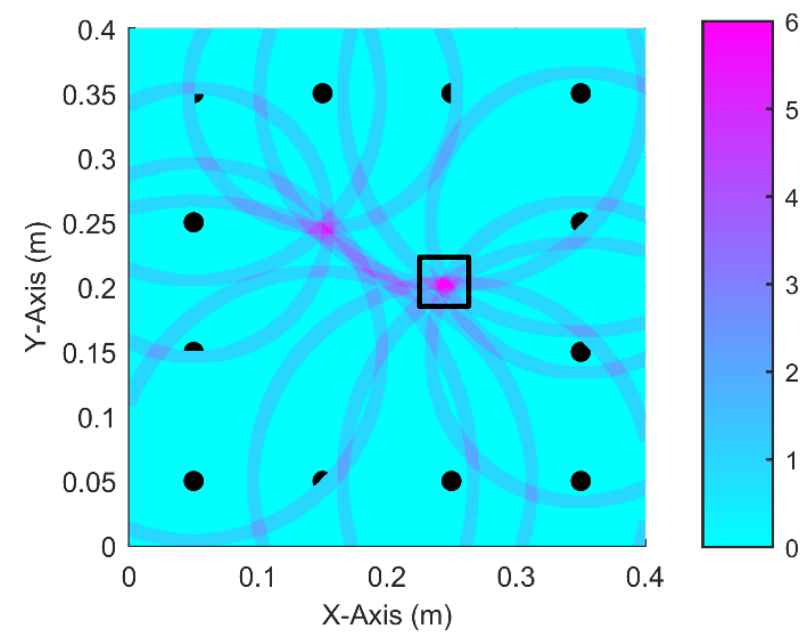

(a) 


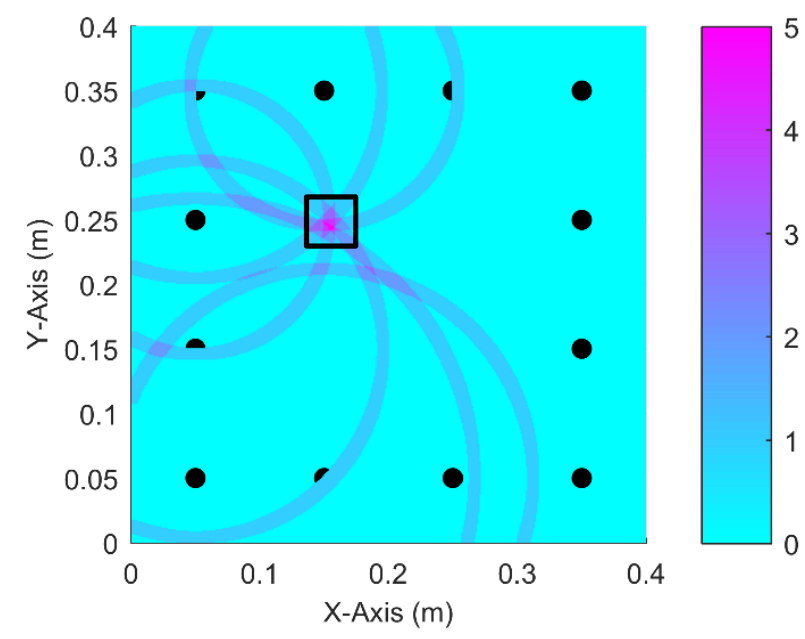

(b)

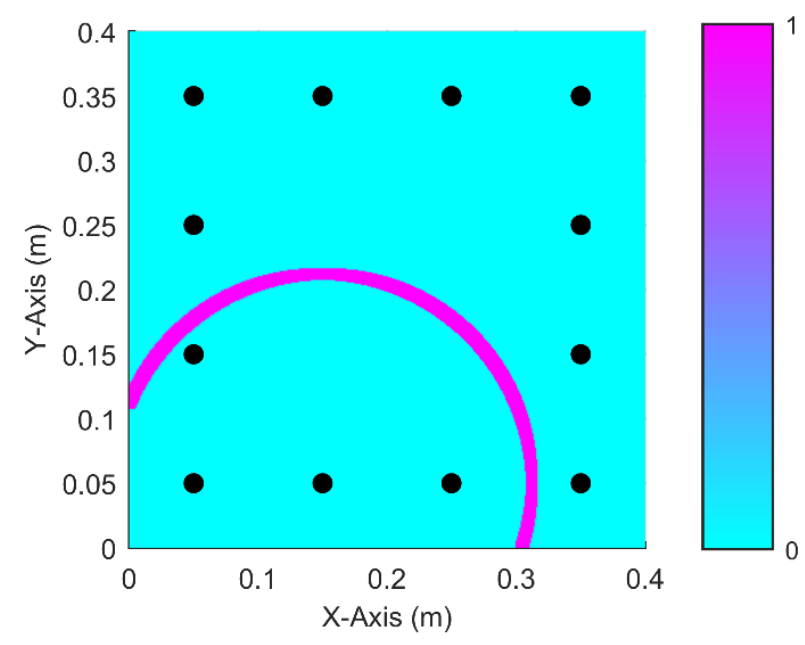

(c)

Figure 19 Number of the detection loci for (a) the first tip, (b) the second tip and (c) the third tip of a kinked crack. The true locations of the crack tips are $(0.15 \mathrm{~m}, 0.25 \mathrm{~m}),(0.2$ $\mathrm{m}, 0.2 \mathrm{~m})$ and $(0.25 \mathrm{~m}, 0.2 \mathrm{~m})$.

Figure 19 shows the result of the initial triangulation for a kinked crack. Based on initial estimates of the locations of the crack tips, it is identified that Transducers 4, 5, 6, 7 and 8 are closer to the first crack tip, and Transducers $1,2,10,11$ and 12 to the second. 
Because Transducer 3 takes part in detecting both crack tips, the information it provides is neglected in the MLAT algorithm in order to avoid ambiguity. Also, since the tip, at which the crack kinks, is seen by only one transducer (i.e. Transducer 9), it is not possible to estimate its exact location.

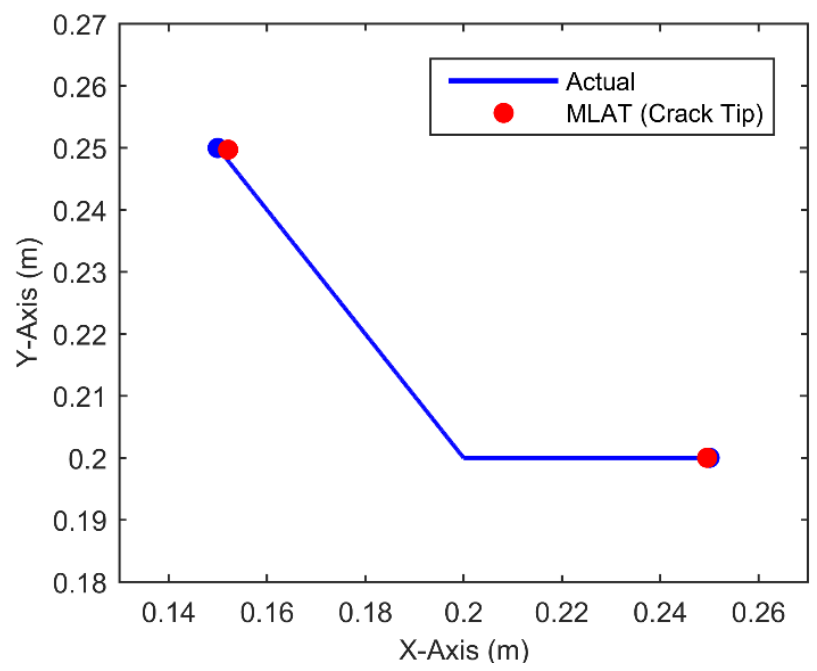

Figure 20 Comparison of the actual and the estimated kinked crack lines.

Based on the known locations of the crack tips and the transducers, it is deduced that the tip, at which the crack kinks, should also be detected by Transducer 10. However, the detection locus of Transducer 10 overshoots the crack line and coincides with the second crack tip. The inclusion of this locus in the MLAT algorithm for the second crack tip is a probabilistic error. However, the influence of this error tip is effectively minimised by the presence of the other detection loci. As displayed in Figure 20, the accuracy of the estimation of the location of the second crack tip is very well retained.

The determination of the location of the tip, at which a shallow kinked crack turns, is not an easy task. As shown by this example, these crack tips will rarely be the ones which most transducers are close to, and hence the loci which detect such a tip would often be mistakenly assigned to other crack tips if there is a slight mismatch between the 
calibration data and the actual velocity of Lamb waves and offset in distance. Also, when there is only one locus left to detect such a crack tip after the elimination process, the wrong assignment of detection loci would not be noticed. Nevertheless, the current technique still shows the capability of detecting the existences of kinked cracks and providing preliminary estimates of their locations.

\section{Experimental Validation}

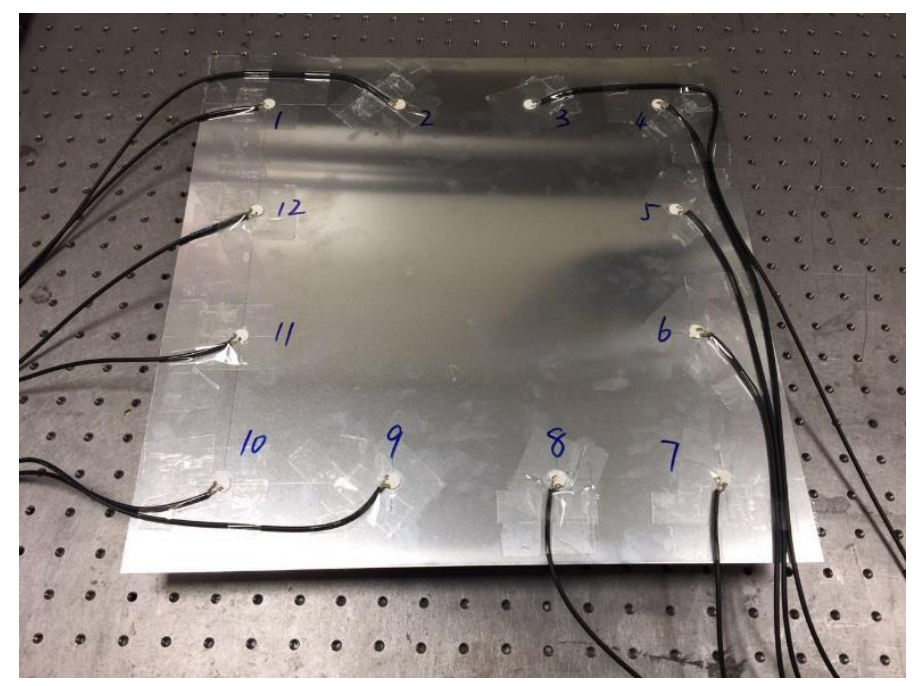

(a)

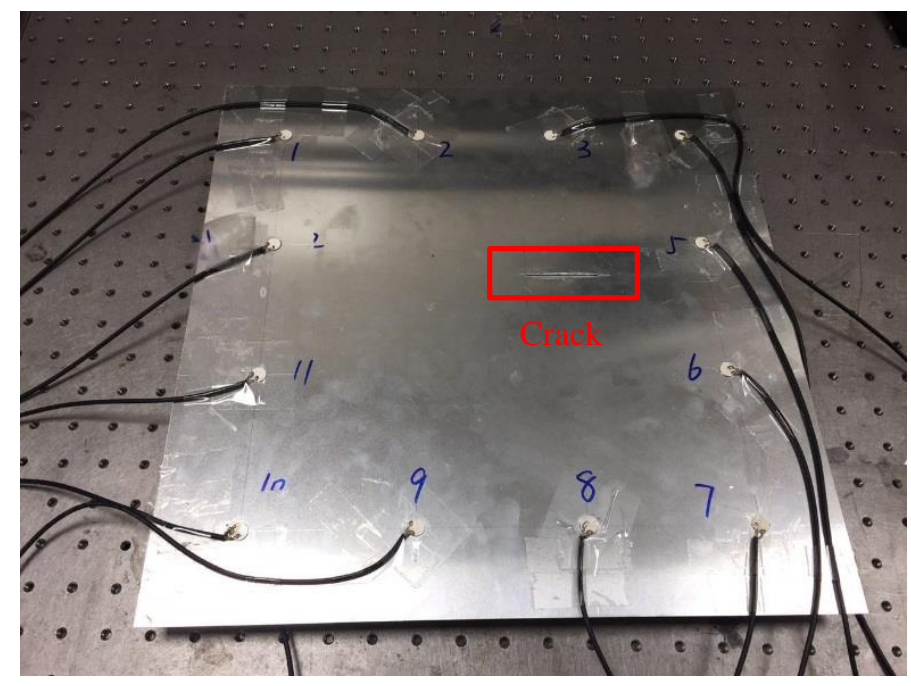

(b) 
Figure 21 (a) The pristine and (b) the damaged specimen used in the experiment.

The crack characterisation technique introduced in this paper has been validated by an experiment. The experimental specimens shown in Figure 21 share the same overall dimension and material properties with the specimens that were used to demonstrate the development of the technique. The piezoelectric transducers are made from Noliac NCE51 ceramic. The excitation signal used is a 5-cycle Hanning windowed sinusoidal toneburst with a central frequency of $380 \mathrm{kHz}$ and a peak amplitude of $12 \mathrm{~V}$. The signal generation and acquisition was done by a TiePie Engineering Handyscope HS3 oscilloscope at a sampling frequency of $50 \mathrm{MHz}$. Through calibration, the velocity of Lamb waves and the offset in distance are found to be $5446.4 \mathrm{~m} / \mathrm{s}$ and $-0.015408 \mathrm{~m}$ respectively.

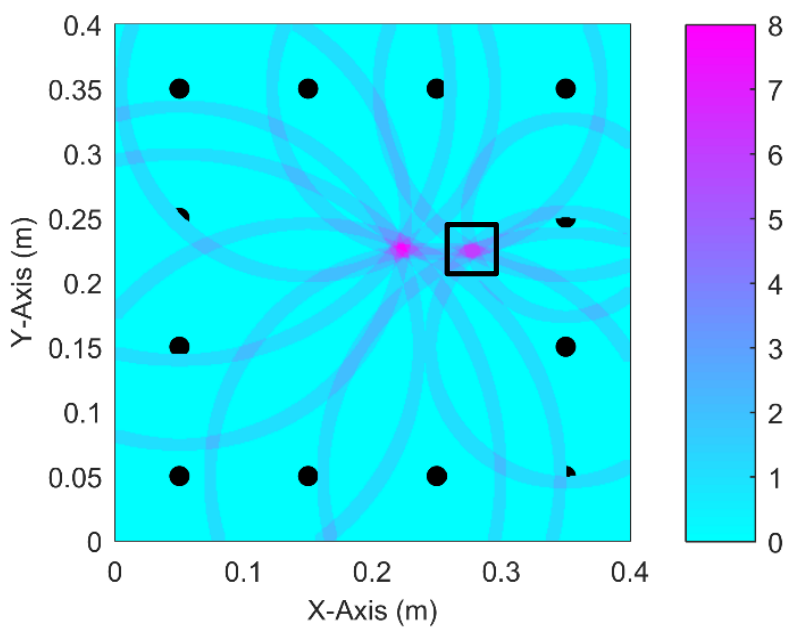

(a) 


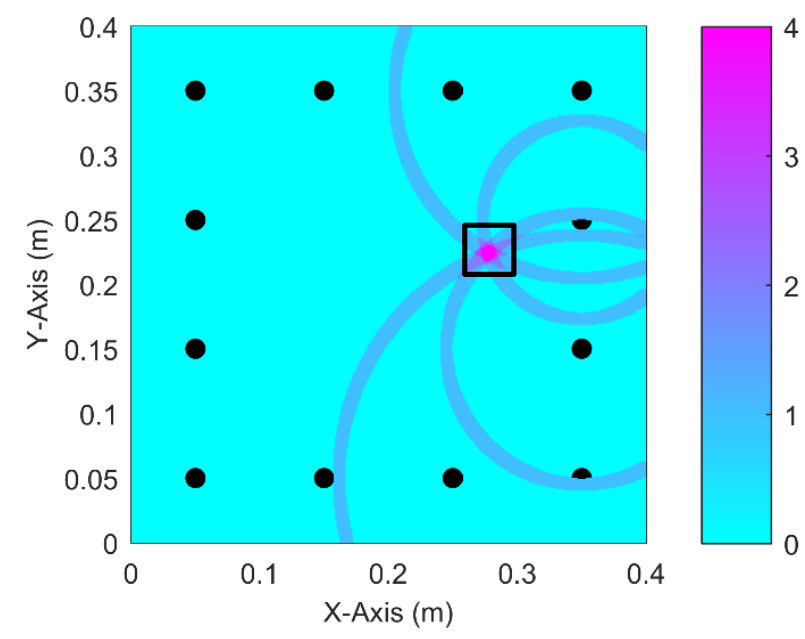

(b)

Figure 22 Number of the detection loci for (a) the first tip and (b) the second tip of the crack on the experimental specimen.

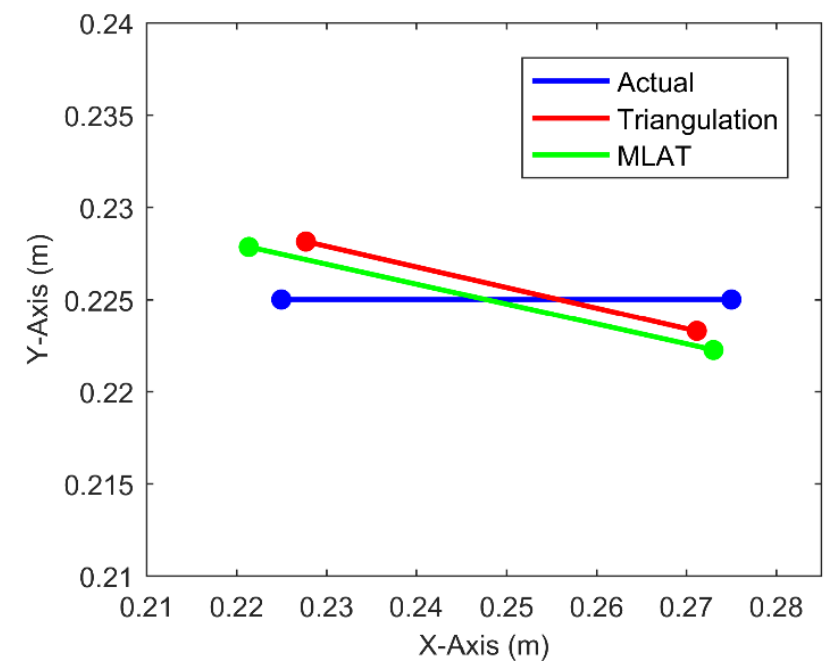

Figure 23 Comparison of the actual and the estimated lines of the crack on the experimental specimen.

Table 4 Estimated parameters of the crack on the experimental specimen. 


\section{Actual Triangulation Error MLAT Error}

$(\%)$

\begin{tabular}{|c|c|c|c|c|c|c|}
\hline Crack Tip 1 & $\mathbf{x}$ & 0.225 & 0.22771 & 5.4286 & 0.22138 & 7.2494 \\
\hline (m) & $\mathbf{y}$ & 0.225 & 0.22814 & 6.2857 & 0.22785 & 5.7023 \\
\hline Crack Tip 2 & $\mathbf{x}$ & 0.275 & 0.27115 & 7.7000 & 0.27301 & 3.9761 \\
\hline (m) & $\mathbf{y}$ & 0.225 & 0.22328 & 3.4500 & 0.22225 & 5.4943 \\
\hline Crack Centre & $\mathbf{x}$ & 0.25 & 0.24943 & 1.1357 & 0.24719 & 5.6128 \\
\hline (m) & $\mathbf{y}$ & 0.225 & 0.22571 & 1.4179 & 0.22505 & 0.10401 \\
\hline Crack Length (m) & & 0.05 & 0.043708 & 12.585 & 0.051939 & 3.8785 \\
\hline Crack Orientation & $\left({ }^{\circ}\right)$ & 0 & 6.3945 & N/A & 6.1877 & N/A \\
\hline
\end{tabular}

The results of the initial triangulation and the MLAT algorithm are illustrated in Figures 22 and 23 and summarised in Table 4. The accuracy of the experimental result is comparable to that demonstrated by the series of numerical studies. The observations that initial triangulations underestimate crack lengths, and that the MLAT algorithm is able to significantly improve initial estimates are also exhibited by the experimental result. The consistency between the numerical and the experiment results, in terms of detection accuracy and detection tendency, is a sound validation of the technique introduced in this paper. 


\section{Conclusion}

In this paper, an automated in situ technique that makes use of permanentally bonded piezoelectric disks to excite Lamb waves for detecting and sizing cracks in plate-like structures is presented. Through the use of the pulse-echo exictation scheme, the technique overcomes the long-standing difficulties asscociated with the full charaterisation of cracks, and owing to the two-stage detection strategy, it is able to output highly accurate results. The development of the technique utilised both analytic models and the FEM. The detection accuracy and the detection tendency of the technique have been fully validated by an experiment. The technique certainly demonstrates the potential for monitoring cracks in critical engineering structures such as aircrafts and pressure vessels. It will help to ensure the safety operation of these structures, and to minimise maintenance costs.

In order to understand the performance parameters and the limitations of the technique, a series of studies that were based on numerically simulated sensor signals have been carried out. The results indicate that the technique is capable of determining the locations and the sizes of most cracks with high levels of accuracy. In particular, the MLAT algorithm plays a crucial role in achieving this. When crack lengths decrease, the frequencies of diagnostic signals would need to increase. The technique also shows the ability to detect and locate kinked cracks.

\section{References}

1. Ihn, J.-B. and F.-K. Chang, Detection and monitoring of hidden fatigue crack growth using a built-in piezoelectric sensor/actuator network: I. Diagnostics. Smart materials and structures, 2004. 13(3): p. 609.

2. Lu, Y., et al., Artificial neural network (ANN)-based crack identification in aluminum plates with Lamb wave signals. Journal of Intelligent Material Systems and Structures, 2009. 20(1): p. 39-49. 
3. Masserey, B. and P. Fromme, In-situ monitoring of fatigue crack growth using high frequency guided waves. NDT \& E International, 2015. 71: p. 1-7.

4. Michaels, J.E., Detection, localization and characterization of damage in plates with an in situ array of spatially distributed ultrasonic sensors. Smart Materials and Structures, 2008. 17(3): p. 035035.

5. Zhao, X., et al., Active health monitoring of an aircraft wing with embedded piezoelectric sensor/actuator network: I. Defect detection, localization and growth monitoring. Smart materials and structures, 2007. 16(4): p. 1208.

6. Sharif-Khodaei, Z. and M. Aliabadi, Assessment of delay-and-sum algorithms for damage detection in aluminium and composite plates. Smart materials and structures, 2014. 23(7): p. 075007.

7. Su, Z. and L. Ye, Fundamental Lamb mode-based delamination detection for CF/EP composite laminates using distributed piezoelectrics. Structural Health Monitoring, 2004. 3(1): p. 43-68.

8. $\mathrm{Yu}, \mathrm{L}$. and V. Giurgiutiu, In situ 2-D piezoelectric wafer active sensors arrays for guided wave damage detection. Ultrasonics, 2008. 48(2): p. 117-134.

9. Pialucha, T., C. Guyott, and P. Cawley, Amplitude spectrum method for the measurement of phase velocity. Ultrasonics, 1989. 27(5): p. 270-279.

10. Bradford, P.W., J. Spilker, and P. Enge, Global positioning system: theory and applications. AIAA Washington DC, 1996. 109.

11. Rose, J.L., Ultrasonic waves in solid media. 1999, Cambridge University Press (New York).

12. Crawley, E.F. and J. De Luis, Use of piezoelectric actuators as elements of intelligent structures. AIAA journal, 1987. 25(10): p. 1373-1385.

13. Lin, X. and F. Yuan, Diagnostic Lamb waves in an integrated piezoelectric sensor/actuator plate: analytical and experimental studies. Smart materials and structures, 2001. 10(5): p. 907.

14. Giurgiutiu, V., Tuned Lamb wave excitation and detection with piezoelectric wafer active sensors for structural health monitoring. Journal of intelligent material systems and structures, 2005. 16(4): p. 291-305.

15. Raghavan, A. and C.E. Cesnik, Finite-dimensional piezoelectric transducer modeling for guided wave based structural health monitoring. Smart materials and structures, 2005. 14(6): p. 1448.

16. Giurgiutiu, V., J. Bao, and W. Zhao, Piezoelectric wafer active sensor embedded ultrasonics in beams and plates. Experimental Mechanics, 2003. 43(4): p. 428-449.

17. Raghavan, A. and C.E. Cesnik, Review of guided-wave structural health monitoring. Shock and Vibration Digest, 2007. 39(2): p. 91-116.

18. Yeasin Bhuiyan, M., Y. Shen, and V. Giurgiutiu, Interaction of Lamb waves with rivet hole cracks from multiple directions. Proceedings of the Institution of Mechanical Engineers, Part C: Journal of Mechanical Engineering Science, 2017: p. 0954406216686996.

19. Rao, J., M. Ratassepp, and Z. Fan, Guided Wave Tomography Based on Full Waveform Inversion. IEEE transactions on ultrasonics, ferroelectrics, and frequency control, 2016. 63(5): p. 737-745. 
20. Huthwaite, P. and F. Simonetti, High-resolution guided wave tomography. Wave Motion, 2013. 50(5): p. 979-993.

21. Bhuiyan, M.Y., Y. Shen, and V. Giurgiutiu, Guided wave based crack detection in the rivet hole using global analytical with local FEM approach. Materials, 2016. 9(7): p. 602.

22. Yang, C., et al., Some aspects of numerical simulation for Lamb wave propagation in composite laminates. Composite structures, 2006. 75(1): p. 267-275.

23. Giurgiutiu, V. and J. Bao. Embedded ultrasonic structural radar for the nondestructive evaluation of thin-wall structures. in Proceedings of the 2002 ASME International Mechanical Engineering Congress. 2002.

24. Lu, Y., et al., Quantitative assessment of through-thickness crack size based on Lamb wave scattering in aluminium plates. Ndt \& E International, 2008. 41(1): p. 59-68. 